\title{
Ductography and Galactomosynthesis in the 21st Century: Role of Imaging in Identifying Endoductal Breast Lesions and in Pre-Surgical Planning
}

\author{
Silvia Bagnera ${ }^{1}$, Erika Giovanna Comello², Carla Berrino ${ }^{1}$, Giulia Berrino ${ }^{1}$, Aurelio Santi Motta ${ }^{1}$, \\ Roberta Ferraro ${ }^{1}$, Sebastiano Patania ${ }^{1}$
}

${ }^{1}$ Department of Diagnostic Imaging A.S.L. TO4 (Ciriè Community Hospital, Ivrea Community Hospital, Chivasso Community Hospital) and Breast Screening Unit, Turin, Italy

${ }^{2}$ Department of Pathology, Ivrea Community Hospital (A.S.L. TO4), Turin, Italy

Email: *sbagnera@aslto4.piemonte.it

How to cite this paper: Bagnera, S., Comello, E.G., Berrino, C., Berrino, G., Motta, A.S., Ferraro, R. and Patania, S. (2021) Ductography and Galactomosynthesis in the 21st Century: Role of Imaging in Identifying Endoductal Breast Lesions and in Pre-Surgical Planning. Open Journal of Radiology, 11, 55-69. https://doi.org/10.4236/ojrad.2021.113006

Received: March 25, 2021

Accepted: July 2, 2021

Published: July 5, 2021

Copyright () 2021 by author(s) and Scientific Research Publishing Inc. This work is licensed under the Creative Commons Attribution International License (CC BY 4.0).

http://creativecommons.org/licenses/by/4.0/ (c) (i) Open Access

\begin{abstract}
Objective: To evaluate the role of invasive imaging in the identification and pre-surgical localization of endoductal breast lesions. Methods: We retrospectively evaluated cytological outcomes, non-invasive/invasive breast imaging obtained between January 2016 and December 2019 in women with pathological nipple discharge (PND). We analysed sensitivity, specificity, positive predictive value and negative predictive value. We also evaluated the advantages of a pre-surgical radiological study using an endoductal contrast medium (with 3D-technique, in young women with dense breasts). Results: A total of 286 women with PND underwent cytological examination, mammography and/or breast ultrasound. When the cytological outcome was reported as "negative" (66.78\%) in agreement with negative noninvasive imaging, patients were sent to follow up. Patients with cytological outcomes defined as "bloody with papillary clusters" (29.37\%) "bloody not associated to cytological modifications" (2.44\%), or "atypical/suspected" for malignant (1.39\%) underwent an invasive procedure. Sensitivity, specificity, positive predictive value and negative predictive value were, respectively: $92.63 \%, 100 \%, 100 \%$ and $96.46 \%$ for cytological examination; $64.28 \%, 96.95 \%, 60 \%$ and $97.44 \%$ for mammography; $41.11 \%, 97.44 \%, 88.09 \%$ and $78.27 \%$ for ultrasound; $93.68 \%$, $100 \%, 100 \%$ and $96.95 \%$ for invasive procedures. Post-surgical histological outcomes confirmed the diagnosis. Conclusion: In absence of a standard diagnostic algorithm, we recommend invasive procedures to identify intraductal breast lesions and for preoperative planning. Digital imaging and new technologies such as $3 \mathrm{D}$-tomosynthesis lead to a renaissance of breast inva-
\end{abstract}


sive imaging; they are confirmed to be an essential diagnostic modality for preoperative planning, to define localization and extension of multiple coexisting endoductal lesions.

\section{Keywords}

Breast, Ductography, Galactomosynthesis, Pathological Nipple Discharge, Invasive Imaging

\section{Introduction}

Pathological nipple discharge (PND) such as single duct monolateral bloody fluid, is a relatively common symptom: as many as one third of all women spontaneously present this at some point in their life, generating great anxiety and discomfort [1]. In literature, the most common cause of nipple discharge is benign diseases such as intraductal lesion. However, PND can also be the first sign of underlying malignancy (in a small percentage, with an incidence varying in literature from $5 \%$ to $23 \%$ ) [2] [3].

The diagnostic protocol of PND remains controversial. According to the American College of Radiology's appropriateness criteria, diagnostic mammography is the standard initial step in patients 30 years of age or older [4]. Since mammography has a low sensitivity $(7 \%$ - 10\%) for the detection of malignancy in this setting, ultrasound with high-frequency transducers $(10-18 \mathrm{MHz}) \mathrm{com}$ plements diagnostic evaluation by allowing for a detailed evaluation of the sub-areolar ducts (with sensitivities and specificities of up to 91\%) and by providing guidance for subsequent needle biopsy, if needed [5] [6] [7].

The cytological examination scores poorly in accuracy, is not helpful in localizing a tumor, and has not been found to possess any significant complementary diagnostic value [8] [9].

In the setting of a negative mammogram and ultrasound, invasive imaging (I.I.) may be performed to evaluate the etiology of PND. Ductography is a technique in which iodinated contrast is injected by cannulating the discharging duct in order to detect lesions causing nipple discharge. It helps in locating the mass and gives useful information for surgical approach.

The first such procedure was described in the 1930s by E. Ries [10]. The contrast medium initially used was lipiodol; the first useful diagnostic results were obtained in the 1940s when water-soluble contrast media began to be used. In the 1960s and 1970s the use of ductography was tested in larger studies as routine clinical practice [11] [12]. For decades, ductography (with a sensitivity and specificity of up to $95 \%$ ) was the only imaging procedure capable of showing the mammary ducts [13].

Ductography has profited from advances in X-ray technology (as the full-field digital mammography, FFDM), the use of local anesthetics, and the development 
of improved non-ionic contrast media. In particular, performing ductography with DBT-technique can increase the detection rate of lesions removing overlapping artifacts [14].

Magnetic resonance imaging (MRI) is a highly sensitive technique in the detection of breast malignancies [15] [16]. However, the use of breast MRI to solve clinical problems in the setting of PND is still limited. Some of the reasons include the cost of MRI, the long duration of the exam, poor accessibility and patient factors such as severe claustrophobia, allergy to contrast media, compromised renal function, severe obesity and implantable devices not compatible with MRI [17]. Since many of these factors do not apply to ductography, it remains an attractive option.

In our study, we aimed to investigate the outcomes of patients evaluated for PND discharge in the pre-surgical setting and to determine the utility of I.I. in multimodality approach.

\section{Materials and Methods}

We retrospectively evaluated all diagnostic investigations carried out between January 2016 and December 2019 in women with unilateral PND. Type of nipple discharge, mammographic/sonographic findings, histopathological outcomes and final diagnosis were recorded.

\subsection{Cytological Nipple Secretion Examination}

All women underwent cytological examination of the discharge. The cytological outcome was reported as "bloody with papillary clusters" suggesting intraductal papillomatosis, "bloody, not associated to cytological modifications", "atypi$\mathrm{cal} /$ suspected for malignant lesion" or "negative". With the exception of women with negative cytological outcomes, all others underwent I.I.

\subsection{Mammography and/or Ultrasound}

In patients of 30 years of age or older, digital mammography was performed as a primary evaluation. The mammography systems used for investigation were: two Mammomat 3000 Siemensand one Senographe 800T GE Healthcare adapted with indirect digital modality (from 2016 to 2017); three Amulet Innovality Fujifilm (from January 2018).

Bilateral whole breast and axillary-region US-imaging was carried out in all women, using high-resolution linear-array transducer (12 - $15 \mathrm{MHz}$; using as ultrasound machine a Mylab Classe C Esaote).

Positive findings on ultrasound included masses or dilated ducts. Any solid focalities was subjected to ultrasound-guided TruCut core biopsy (CB, with a 14 $\mathrm{G}$ needle). In some cases, at the end of the biopsy procedure, a nonmagnetic echo-visible radiopaque intralesional clip was placed. In the presence of lesions visible only on mammography, biopsy analysis was performed with vacuum assisted method (VABB). 


\subsection{Ductography}

The ductography was carried out by cannulating the single secreting duct with a dedicated blunt 30-gauge cannula; the particular shape of the tip prevents unwanted needle deviations. Before proceeding with the cannulation, we applied a lidocaine/prilocaine cream on the nipple to facilitate the procedure and prevent patient discomfort.

After the cannulation, a contrast agent (iodinate contrast media $300 \mathrm{mg} / \mathrm{ml}$ ) was injected. Cranio-caudal/lateral projections and mammograms done with microfocus magnification view (aimed at evaluating of specific areas of the breast) were performed. Two experienced breast radiologists (respectively with over 11 and 22 years of experience) evaluated and classified the galactograms by consensus.

\subsection{Ductography with Tomosinthesis Technique (Galactomosynthesis)}

From January 2018 on, ductography with Digital Breast Tomosynthesis (DBT, a 3D-tecnique) was applied to young patients under the age of 45 and with breast dense tissue classified with visual way as " $\mathrm{C}$ " - i.e. heterogeneously dense (which could obscure the detection of small masse) - or " $\mathrm{D}$ "-i.e. extremely dense-by the American College of Radiology (ACR) Breast Imaging-Reporting and Data System (BI-RADS) Density Descriptions [18]. Synthetic digital 2D full-field mammograms were generated from the DBT-data using the process software systems and the assessment criteria were the same of traditionally ductography.

\subsection{MRI}

MRI was performed as a problem-solving ancillary examination selectively (i.e. when invasive/non invasive imaging was non-informative or if the extent of the disease remained unclear). The MRI examinations were performed with a $1.5 \mathrm{~T}$ MRI scanner (Achieva D-Stream, Philips).

\subsection{Diagnostic Process and Final Definition with Follow-Up or Surgical Planning for Selective Exeresis}

In the presence of a "negative" cytological outcome with mammographic and ultrasound negative imaging the women were sent to clinical-radiological follow-up. In the presence of a "bloody" or "atypical/suspected" cytological outcome the protocol entailed invasive imaging, sometime with the addition of biopsy.

When the ductography showed the presence and location of single or multiple endoductal lesions and when micro-histological outcomes were "B3-lesions with atypia" or "B4/B5-suggesting breast heteroplasia" such as in situ carcinoma (DCIS) and invasive carcinoma (IC), the women were sent for surgery.

The ductography report included: the distance between the endoductal lesion and the nipple areola-complex (NAC), the overall extention of papillomatosis 
(when present), and the distance between the endoductal finding and any further associated lesions.

The postoperative histological outcome was used as gold standard.

\section{Results}

From January 2016 to December 2019, 286 women were investigated at our centre for PND. The median patient age was 55 years (range: 22 - 82 years).

All women underwent cytological examination of the discharge. A mammography with associated ultrasound evaluation was done in all cases except in the 7 young women (under 30 years of age) for whom an ultrasound study was performed as the only first examination.

The cytological outcome was reported as "negative" in 191 cases (66.78\%); all of these showed negative mammographic and ultrasound imaging, therefore no other investigations were performed and the patients were all sent to follow up without finding pathological lesions at the next checkup (performed after two years in $80 \%$ of the cases). The other cytological results were defined as "bloody with papillary clusters" in 84 patients (29.37\%), "bloody, not associated to cytological modifications" in 7 cases (2.44\%), or "atypical/suspected" for malignant lesion in 4 cases (1.39\%).

Considering the cytological outcome reported as "bloody with papillary clusters" suggesting intraductal papillomatosis (84 cases), a retrospective analysis showed that the mammography was negative in $80.95 \%$ of cases. Only in $10.71 \%$ of cases did a mammography identify a BI-RADS R3 non-specific finding (subjected to biopsy, as shown in Table 1). The ultrasound was always performed and was negative in $63.09 \%$. The ultrasound imaging revealed the presence of a ductal ectasia associated with iso-/hypoechoic extraductal focality (in $23.80 \%$ of cases) or with endoductal solid lesion (in $10.71 \%$ of cases). In two cases ductal ectasia was associated with both solid extraductal and endoductal lesions. All the solid extraductal masses were biopsied and a nonmagnetic echo-visible radiopaque clip was placed into the lesion in $22.72 \%$ of cases.

All 84 cytological outcomes reported as "bloody with papillary clusters" were completed with I.I.: 77 with ductography and 7 with DBT-technique (the latter in young women with high breast density classified as "D" by the ACR BI-RADS Density Descriptions), to reduce tissue overlapping artifacts.

All women well tolerated the invasive procedures. There were no intra-procedural difficulties nor need for re-injection of the endoductal contrast medium. We took care to remove all air bubbles that may have mimicked intraductal lesions in the contrast column so as to avoid a false "cut-off" sign.

As shown in Table 1, the ductography identified: 61 cases of ductal ectasia with multiple fine endoductal parietal irregularities (suggesting "papillomatosis", as identifiable in Figure 1 and Figure 2); 19 cases of ductal ectasia with "single intraductal papilloma" visible as gross endoductal filling defect (with "cut-off" sign), as viewable in Figure 3 and Figure 4. 
Table 1. Cases of cytological outcome reported as "bloody with papillary clusters" suggesting intraductal papillomatosis: correlation with mammography $(\mathrm{Mx})$ and/or breast ultrasound features (US), invasive imaging (DUCTO), pre- and post-surgical histological outcomes.

\begin{tabular}{|c|c|c|c|c|c|c|}
\hline $\begin{array}{l}\text { Cytol. } \\
\text { Outcome }\end{array}$ & $\begin{array}{l}\text { Type of } \\
\text { Imaging }\end{array}$ & \multicolumn{3}{|c|}{ Imaging Outcome } & Biopsy Outcome & $\begin{array}{c}\text { Post Surgical } \\
\text { Hystol. } \\
\text { Outcome }\end{array}$ \\
\hline \multirow{12}{*}{$\begin{array}{c}84 \\
\text { Bloody } \\
\text { with } \\
\text { papillary } \\
\text { clusters }\end{array}$} & \multirow{5}{*}{ MX } & NEG & & 68 & ------ & \multirow{12}{*}{$\begin{array}{l}84 \text { Papillary } \\
\text { Lesion }\end{array}$} \\
\hline & & \multirow{4}{*}{ POS. } & \multirow{3}{*}{ BIRADS R3 } & 5 Micro & 5 VABB: $\mathrm{B} 2$ & \\
\hline & & & & 3 Op. & 3 CB: B3 & \\
\hline & & & & 1 P.T & 1 VABB: $\mathrm{B} 2$ & \\
\hline & & & BIRADS R4/R5 & ------- & ------- & \\
\hline & \multirow{4}{*}{ US } & NEG & \multirow{3}{*}{ BIRADS U3 } & 53 & ------ & \\
\hline & & & & $9^{*}$ & ------- & \\
\hline & & POS. & & $22^{* *}$ & $22 \mathrm{CB}: 17 \mathrm{~B} 3+5 \mathrm{~B} 2$ & \\
\hline & & & BIRADS U4/U5 & ------- & ------ & \\
\hline & \multirow{3}{*}{ DUCTO } & NEG & & 4 & $-\cdots--$ & \\
\hline & & \multirow{2}{*}{ POS. } & D.E. + M.E.I & $61^{* * *}$ & ----- & \\
\hline & & & D.E. + "cut-off" sign & $19^{* * * *}$ & $-\cdots--$ & \\
\hline
\end{tabular}

Legend: Op: Opacities; Micro: Microcalcifications; P.T.: Parenchymal Thickening. VABB: Vacuum Assisted Breast Biopsy (with needle 10 Gauge; CB: Core Biopsy (Trucut with needle 14 Gauge); B2: "benign lesion without atypia and associated with dystrophic fibrosclerosis phenomena"; B3: "papillary lesion with atypia"; ${ }^{*}$ ductal ectasia (D.E.) + endoductal solid lesions; ${ }^{* *}$ ductal ectasia (D.E.) + extraductal solid lesions; ${ }^{* * *}$ ductal ectasia (D.E.) + multiple endoductal irregularity (M.E.I.); ${ }^{* * *}$ ductal ectasia (D.E.) with "cut-off" sign.
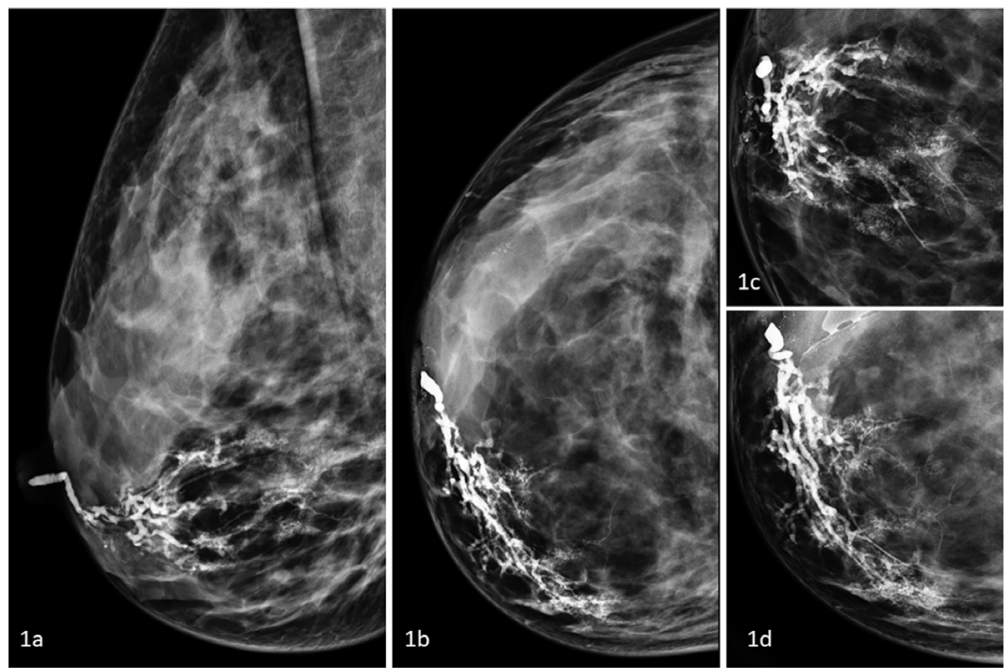

Figure 1. 31-year-old woman with unilateral mono-orificial bloody nipple discharge classified as "bloody with papillary clusters" at the cytological examination and with ultrasound imaging suggesting lobar ductal ectasia, subjected to invasive procedure. After administration of endoductal contrast medium, identification of ductal ectasia with millimeter multiples peri-parietal filling defects both at the (a) medio-lateral-oblique and (b) cranio-caudal projections and in the magnification view (c) and (d), indicating "intraductal papillomatosis" involving the entire lobe of the infero-medial right breast region.

These cases highlight the great clinical benefit of using invasive imaging: without the use of ductography these cases of papillomatosis (post-operative 
confirmed) would not have been identified. The women would have risked being sent for clinical follow-up (in some cases with the support of a negative biopsy outcome) and they risked not receiving surgical treatment. Thanks to I.I., a targeted intervention was made, without complications.
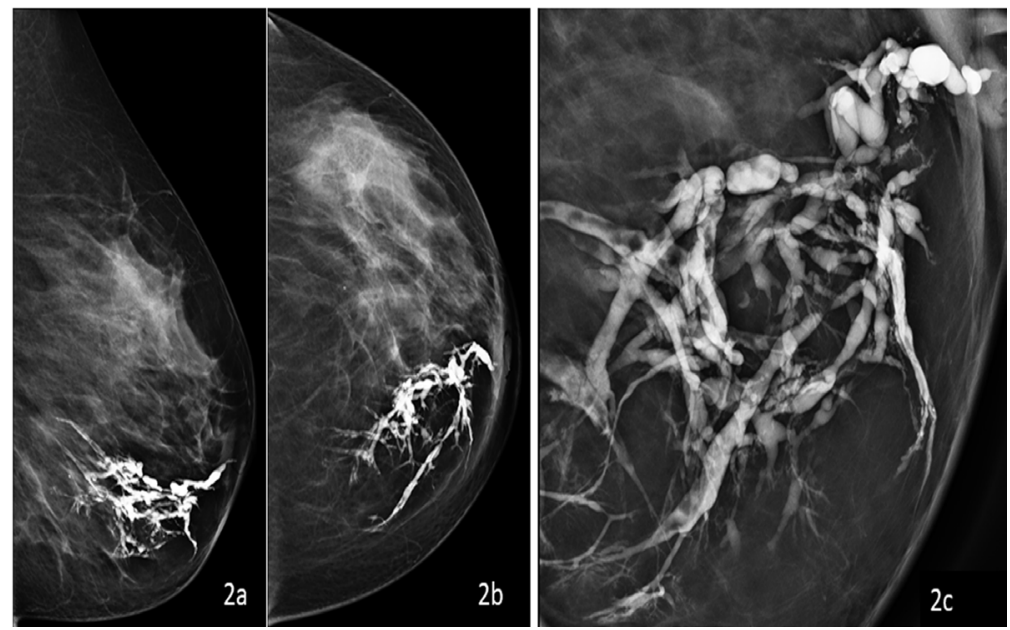

Figure 2. 40-year-old woman with unilateral mono-orificial bloody nipple discharge classified as "bloody with papillary clusters" at the cytological examination (with negative mammography and ultrasound imaging suggesting lobar ductal ectasia), subjected to invasive procedure. After administration of endoductal contrast medium, identification of ductal ectasia with millimeter multiples peri-parietal filling defects both at the (a) medio-lateral-oblique and (b) cranio-caudal projections and in the magnification view (c), indicative for "intraductal papillomatosis" involving the entire lobe of the infero-medial left breast region.
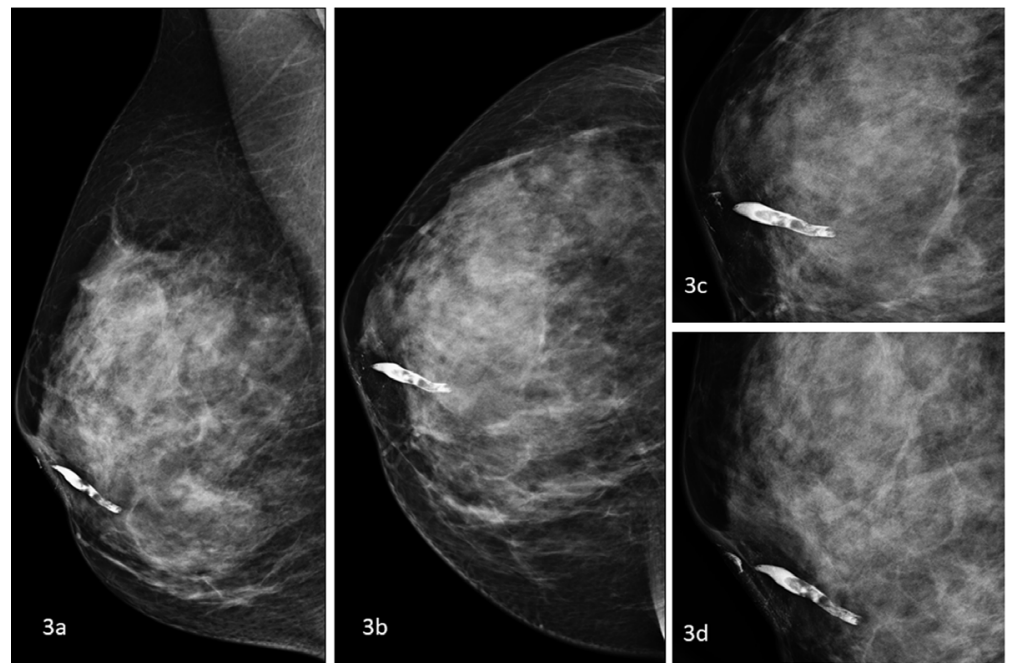

Figure 3. 37-year-old woman with unilateral mono-orificial bloody nipple discharge classified as "bloody with papillary clusters" at the cytological examination and with negative ultrasound imaging, subjected to galactomosynthesis. At the syntetical digital 2D mammograms (a) medio-lateral-oblique and (b) cranio-caudal projections, generated with this technique, after introduction of endoductal contrast medium, identification of retroareolar ductal ectasia (towards the lower quadrants) with solid endoductal lesions and visualization of "cut off sign", better appreciated in the magnification view (c) and (d). 

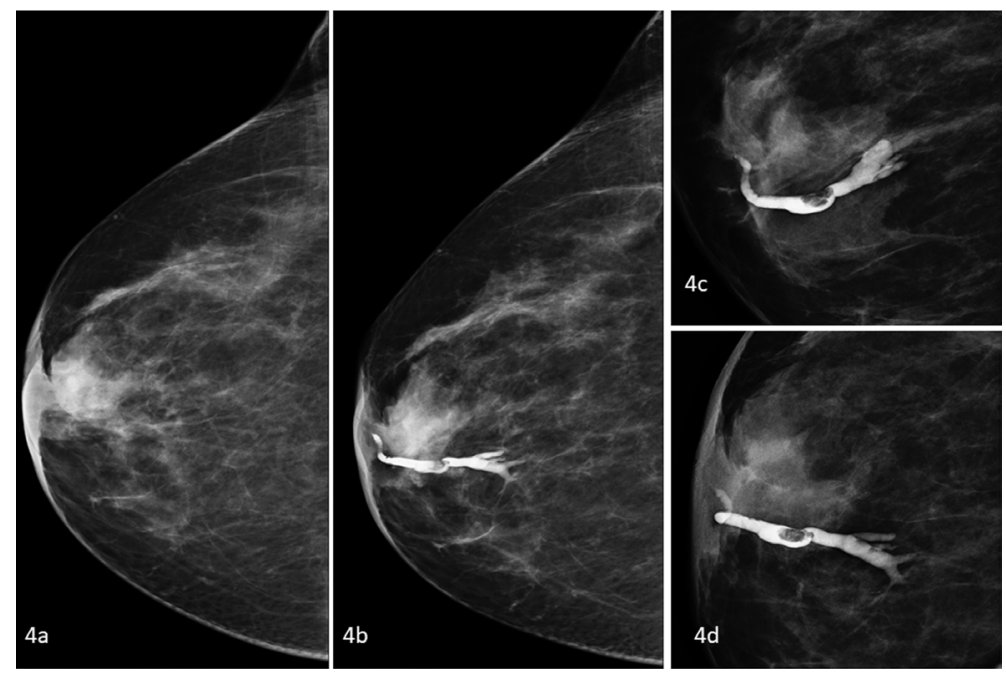

Figure 4. 45-year-old woman with unilateral mono-orificial bloody nipple discharge classified as "bloody with papillary clusters" at the cytological examination and with negative mammography/ultrasound imaging, subjected to ductography. Cranio-caudal mammogram projection showing absence of pathological findings (a); the same view after administration of endoductal contrast medium (b), identification of ductal ectasia with retroareolar gross endoductal filling defect suggesting "intraductal papilloma" and posterior identification of multiple dilated ducts, better appreciated in the magnification view (c) and (d).

Only in 4 cases I.I. had identified ductal ectasia with homogeneous opacification of one or more ectasic ducts, without evident filling defects. Even in these cases (considered to be false negatives), the ductography still made an important contribution by modifying the surgical approach and allowing the targeted removal of only the ectasic ducts identified in the galactograms in the specific breast quadrant.

As shown in Table 2, the 7 cytological outcomes reported as "bloody, not associated to cytological modifications" presented in 5 cases negative mammography with ultrasound identification of ductal ectasia associated with iso-/hypoechoic extraductal focality, all subjected to $\mathrm{CB}$ and resulted as 4 papillary lesion and 1 DCIS at the post-surgical histological outcomes. In all these cases, I.I. identified ductal ectasia with associated multiple endoductal irregularities (as in Figure 5), precisely defining the overall extension of the pathology responsible for the secretion. In 2 cases, mammography showed BI-RADS 3 microcalcifications (subjected to VABB, with outcome of 1 DCIS and 1 IC). In these two cases, the ultrasound showed only a nonspecific ectasia while more ectasic ducts appeared in the ductography (however with no evident filling defects); post-operative outcomes confirmed 1 DCIS and 1 IC associated with "multiple areas of intraductal papillomatosis" extended up to the NAC.

These cases are explanatory of the added value of invasive imaging: the panoramic study of the ductal system of the breast. In the absence of I.I., these women would have undergone focal tumorectomy, with the risk of persistent bloody secretion after surgery and/or an increased risk of loco-regional recurrence dis- 
ease.

As shown in Table 3, the four cases with "atypical/suspected" cytological outcome presented positive mammographic imaging for BI-RADS R5 suspicious lesions, all identified as hypoechoic masses (classified as two BI-RADS U5 and two as BI-RADS U3), submitted to CB (all post-surgical confirmed as 3 DCIS and 1 IC). At the ductography, ductal ectasia associated with endoductal multiple irregularity (in 3 cases) and ductal ectasia with "cut-off" sign (in 1 case) was detected, highlighting the multifocal nature of heteroplasia (as shown in Figure 6).

Table 2. Cases of cytological outcome reported as "bloody not associated to cytological modification": correlation with mammography $(\mathrm{Mx})$ and/or breast ultrasound features (US), invasive imaging (DUCTO), pre- and post-surgical histological outcomes.

\begin{tabular}{|c|c|c|c|c|c|c|}
\hline $\begin{array}{c}\text { Cytol. } \\
\text { Outcome }\end{array}$ & $\begin{array}{l}\text { Type of } \\
\text { Imaging }\end{array}$ & & Imaging Outcome & & Biopsy Outcome & $\begin{array}{c}\text { Post Surgical } \\
\text { Hystol. } \\
\text { Outcome }\end{array}$ \\
\hline \multirow{9}{*}{$\begin{array}{c}7 \\
\text { Bloody } \\
\text { not } \\
\text { assoc. } \\
\text { to } \\
\text { cytol. } \\
\text { modif. }\end{array}$} & \multirow{3}{*}{ MX } & NEG & & 5 & ------- & \multirow{9}{*}{$\begin{array}{c}4 \text { Papillary } \\
\text { Lesion } \\
+ \\
1 \mathrm{IC} \\
+ \\
2 \text { DCIS }\end{array}$} \\
\hline & & \multirow{2}{*}{ POS. } & BIRADS R3 & 2 Micro & 2 VABB: 1 CDIS/CI & \\
\hline & & & BIRADS R4/R5 & ------ & ------- & \\
\hline & & NEG & & 2 & ----- & \\
\hline & US & POS. & BIRADS U3 & $5^{\star *}$ & 5 CB: 5 B3 & \\
\hline & & & BIRADS U4/U5 & ------ & ------ & \\
\hline & \multirow{3}{*}{ DUCTO } & NEG & & 2 & ------- & \\
\hline & & \multirow{2}{*}{ POS. } & D.E. + M.E.I & $5^{* * *}$ & ------- & \\
\hline & & & D.E. + “cut-off” sign & --- & ------- & \\
\hline
\end{tabular}

Legend: See previous description.

Table 3. Cases of cytological outcome reported as "atypical/suspected": correlation with mammography ( $\mathrm{Mx})$ and/or breast ultrasound features (US), invasive imaging (DUCTO), pre- and post-surgical histological outcomes.

\begin{tabular}{|c|c|c|c|c|c|c|}
\hline $\begin{array}{l}\text { Cytol. } \\
\text { Outcome }\end{array}$ & $\begin{array}{c}\text { Type } \\
\text { of } \\
\text { Imaging }\end{array}$ & & Imaging Outcome & & $\begin{array}{l}\text { Biopsy } \\
\text { Outcome }\end{array}$ & $\begin{array}{c}\text { Post Surgical } \\
\text { Hystol. } \\
\text { Outcome }\end{array}$ \\
\hline \multirow{9}{*}{$\begin{array}{l}4 \text { Atypical/ } \\
\text { Suspected }\end{array}$} & \multirow{3}{*}{ MX } & NEG & & 0 & ------ & \multirow{9}{*}{$\begin{array}{c}3 \text { DCIS } \\
+ \\
1 \mathrm{IC}\end{array}$} \\
\hline & & & BIRADS R3 & ------ & ------ & \\
\hline & & POS. & BIRADS R4/R5 & $\begin{array}{l}2 \text { Micro } \\
2 \mathrm{Op} .\end{array}$ & ----- & \\
\hline & & NEG & & 0 & ------ & \\
\hline & US & & BIRADS U3 & $2^{\star *}$ & $\begin{array}{c}2 \text { CB: } 2 \text { B5 } \\
\text { (2 DCIS) }\end{array}$ & \\
\hline & & PUS. & BIRADS U4/U5 & $2^{* *}$ & $\begin{array}{c}2 \text { CB: } 2 \text { B5 } \\
\text { (1CDIS E } 1 \text { IC) }\end{array}$ & \\
\hline & \multirow{3}{*}{ DUCTO } & NEG & & 0 & ------- & \\
\hline & & \multirow{2}{*}{ POS. } & D.E. + M.E.I & $3 * * *$ & ------ & \\
\hline & & & D.E. + “cut-off” sign & $1^{\star * \star *}$ & ------ & \\
\hline
\end{tabular}

Legend: See previous description. 

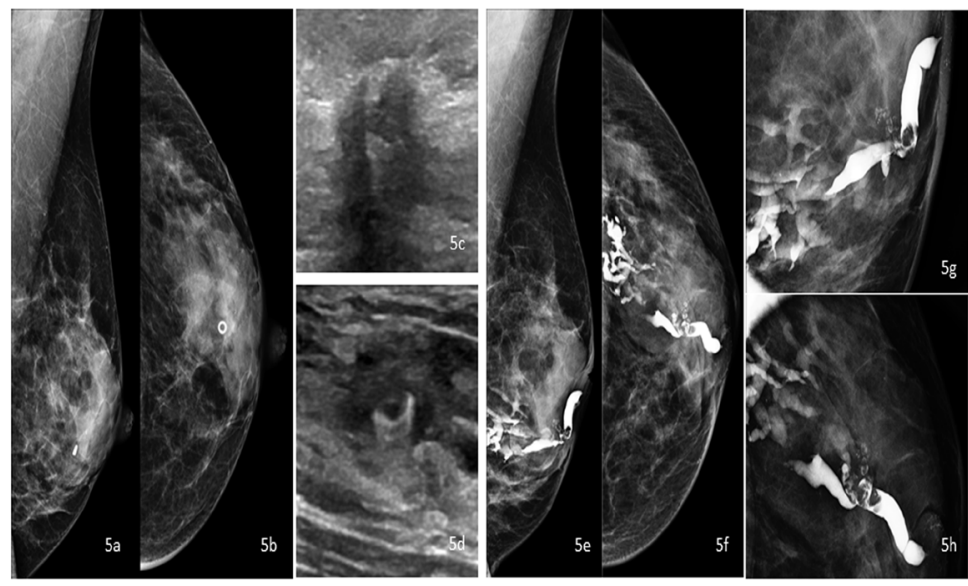

Figure 5. 50-year-old woman with unilateral mono-orificial bloody nipple discharge classified as "bloody, not associated to cytological modification" at the cytological examination. The imaging study was characterized by negative mammography (as shown in radiograms (a) and (b)) with ultrasound identification of ductal ectasia associated to an hypoechoic extraductal focality (c) subjected to needle biopsy (with histological outcome of B3 as papillary lesion) and marked with nonmagnetic echo-visible radiopaque clip (d). At the ductogalactography imaging (e)-(h): identification of a main retroareolar duct ectasia in the infero-lateral left breast region associated with the presence of a gross endoductal filling defect (about $11 \mathrm{~mm}$ in diameter, placed at a distance of $12 \mathrm{~mm}$ from the base of the nipple) suggesting "intraductal papilloma"; visualization also of a concomitant multiple posterior ducts ectasia associated with numerous endoductal filling defects (some with "cut off sign"). Post-operative outcomes confirmed previous diagnoses made by multimodal approach.

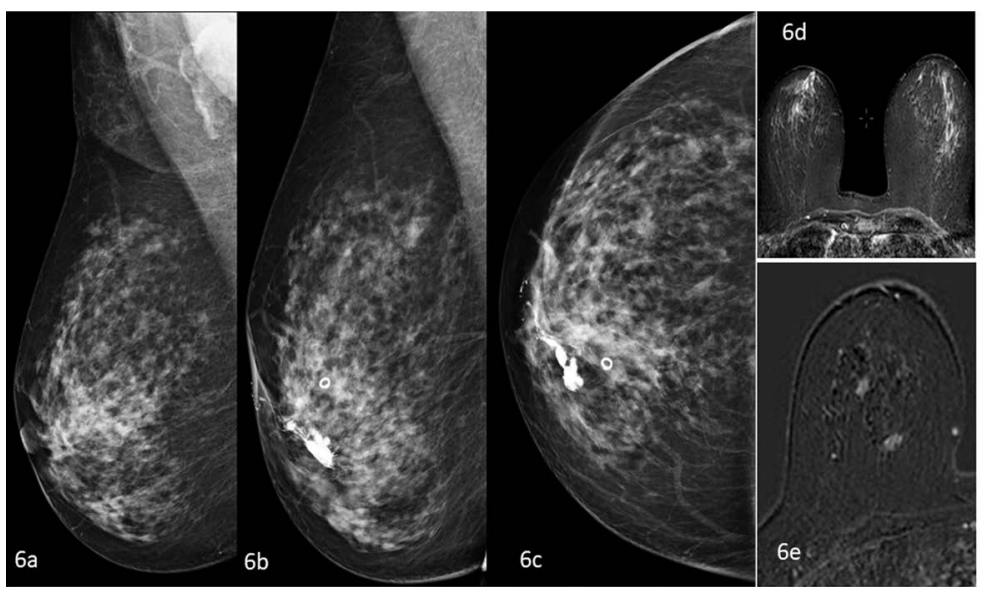

Figure 6. 61-year-old woman with unilateral mono-orificial bloody nipple discharge classified as "atypical/suspected" at the cytological examination. (a) At mammography: appearance in the upper quadrants of millimetric opacity identified as hypoechoic suspicious mass on ultrasound (confirmed by the ultrasound-guided needle biopsy as infiltrative carcinoma and marked with nonmagnetic echo-visible radiopaque clip); (b)-(c) At the lateral and cranio-caudal projections, after introduction of endoductal contrast medium: identification of ductal ectasia with filling defect associated with endoductal multiple irregularity and sudden completed interruption to the passage of the endoductal contrast medium in the medial breast region. (d)-(e) At MRI: confirmation of ductal ectasia (in T2-weighted STIR sequences) and multifocality (as shown in the "subtracted" images, after administration of intravenous contrast medium). 
In all cases, I.I. allowed for a precise definition of the anatomical site of the secreting duct with a precise pre-surgical planning. It was always possible to define the anatomical relationships between the tumor lesion and the endoductal findings visible in the galactograms, often with the finding of multiple quadrants of the same breast affected by pathology.

In the absence of ductography, the protocol might have resulted in "non-free margins" to the pathological report of the operating piece, a risk possibly entailing the need for a second surgery or radiotherapy over-treatment.

MRI was performed only in 6 cases as a supplemental technique before surgery (considering dense breasts and/or family history of breast heteroplasia/BRCA mutations). In all cases the MRI confirmed the ductography, without identification of additional lesions.

We calculated sensitivity, specificity, positive predictive value and negative predictive value of the tests performed with the following results, respectively: $92.63 \%, 100 \%, 100 \%$ and $96.46 \%$ for the cytological examination; $64.28 \%$, $96.95 \%, 60 \%$ and $97.44 \%$ for mammography; $41.11 \%, 97.44 \%, 88.09 \%$ and $78.27 \%$ for ultrasound imaging and $93.68 \%, 100 \%, 100 \%$ and $96.95 \%$ for I.I.

\section{Discussion}

In our study PND is associated with an underlying malignancy (1.39\%, as noted in other studies) but symptoms cause extreme anxiety [19]. A debate about the most effective diagnostic PND algorithm is still ongoing. For Tabar et al., ductography should be performed in all PND patients, in addition to cytological and mammographic examination, while Yoon et al. bemoan the lack of standard guidelines [11] [20].

In our environment, the cytological outcomes are important to guide subsequent investigations. The cases with a "negative" cytological outcome and absence of detectable findings at mammography or ultrasound imaging were sent to follow-up, without finding any pathology in the following years (with an average follow-up of over two years in $80 \%$ of cases). All cases with an "atypical/suspected" cytological outcome correlated with suspicious imaging (both non-invasive and invasive method) were sent to surgery with post-operative confirmation of diagnosis. In cases with "bloody", we proceeded with I.I. even if the result of the mammography/ultrasound imaging was negative and we found a positive imaging for endoductal alterations, such as single/multiple papillary lesion(s) or wall irregularities in $92.63 \%$ of cases.

Thanks to the study of the ductal system through invasive procedures, it was possible to plan a highly selective surgery, allowing the exeresis of the duct responsible for the secretion and sparing the healthy ducts. The added value of I.I. in PND was the possibility of providing the surgeon with accurate information such as the location of the affected duct, the distance from the nipple and the overall extension of the area to be removed.

Ductography might have self-limiting minor complications (such as duct per- 
foration and extravasation of the contrast material) [3]. The technical failure is considered to be a limitation. In the present study, no complications, no adverse effects, and no allergies were observed. The use of lidocaine/prilocaine cream about 50 mins before the examination has been widely used to reduce or eliminate pain [21]. In all our cases, the cannulation of the secreting duct was seamless and rapid.

In our series, in the four cases of pathological PND, ductography highlighted tumor multifocality (with the involvement of a larger mammary area compared to non-invasive imaging evaluation). Thanks to the use of I.I. it was possible to remove, not only the tumor lesion, but also the area of associated papillomatosis often considered as a pre-cancerous lesion.

Ultrasound was more specific (97.44\%) compared to mammography (96.95\%); it is used as an ancillary study to characterize intraductal lesions and to outline the relationship between the mass and the ductal system.

As shown in Table 4 and Table 5, in our study, the sensitivity of I.I. in detecting the etiology of PND was both higher (93.68\%) than what reported in other studies with values as $76 \%, 84.3 \%$ and $77.4 \%$ and when compared with the other noninvasive imaging modalities, with values as $13 \%$ when considering only mammography, $73 \%$ when considering only ultrasound, $85.7 \%$ when considering only MRI and $67.6 \%$ when considering all non invasive modalities $(\mathrm{Mx}+$ US + MRI) [2] [3] [10]. This data point could be attributed to the fact that in our institute invasive procedures are preceded by nipple discharge cytological examinations. This may also be a consequence of the state-of-the-art utilization of ductography by radiologists highly experienced with invasive technique.

We were also able to show that DBT-technique can be used in invasive procedure as an additional option. The cases considered false negatives to ductography (with visualization only of multiple ectasic ducts, without evident filling defects) are probably due to limits of spatial resolution of the imaging currently used; ductography with 3-D method could further reduce false negative cases.

We believe that the standardization of galactogram interpretations (such as the "Galactogram Image Classification System" proposed by Berna-Serna et al.) is not needed; by contrast, we preferred to use a descriptive method allowing the surgeon to have precise anatomical information [10] [22].

EUSOMA guidelines and metanalysis concluded that there is insufficient evidence of benefit to recommend the routine use of MRI in the clinical context of PND [23] [24] [25].

Even if the etiology of PND turns out to be benign, the presence of intraductal lesions at the imaging stage, such as papilloma, requires a surgical intervention. I.I. is a necessary tool for the pre-surgical planning, as it accurately shows the position of the discharging ducts (central/peripheral) as well as the presence of multiple peripheral papillary lesions, and offers the possibility to a focused ductal excision that preserves greater sensation and function, avoids nipple inversion, and decreases the likelihood of seroma formation. 
Table 4. Sensitivity, specificity, positive predictive value (PPV), and negative predictive value (NPV) for ductography. Comparison between our cases and other experiences reported in the literature.

\begin{tabular}{ccccc}
\hline & \multicolumn{4}{c}{ Ductography } \\
\cline { 2 - 5 } & Sensitivity & Specificity & PPV & NPV \\
\hline Our study & $93.68 \%$ & $100 \%$ & $100 \%$ & $96.95 \%$ \\
Baydoun et al. [3] & $76 \%$ & $72 \%$ & $84 \%$ & $61 \%$ \\
Srivasan et al. [2] & $84.3 \%$ & $62 \%$ & $72.2 \%$ & $77 \%$ \\
Istomin et al. [10] & $77.4 \%$ & $75.7 \%$ & $72.7 \%$ & $80 \%$ \\
\hline
\end{tabular}

Table 5. Sensitivity, specificity, positive predictive value (PPV), and negative predictive value (NPV) for ductography in our study compared to the other methods of non-invasive imaging reported in the literature.

\begin{tabular}{ccccc}
\hline & Sensitivity & Specificity & PPV & NPV \\
\hline $\begin{array}{c}\text { Ductography in our study } \\
\text { Non-invasive imaging (only Mx) } \\
\text { in Baydoun et al. study [3] }\end{array}$ & $93.68 \%$ & $100 \%$ & $100 \%$ & $96.95 \%$ \\
$\begin{array}{c}\text { Non-invasive imaging (only US) } \\
\text { in Baydoun et al. study [3] }\end{array}$ & $73 \%$ & $97 \%$ & $89 \%$ & $37 \%$ \\
$\begin{array}{c}\text { Non-invasive imaging (only MRI) } \\
\text { in Istomin et al. study [10] }\end{array}$ & $85.7 \%$ & $71.4 \%$ & $60 \%$ & $90.9 \%$ \\
$\quad$ Mx + US + MRI & & $97 \%$ & $64 \%$ \\
$\quad$ in Srivasan et al. study [2] & $67.6 \%$ & $75 \%$ & $76 \%$ & $66.3 \%$ \\
\hline
\end{tabular}

This study does not intend to modify established practices at other centers in the management of PND, but it wishes to emphasize the usefulness of inexpensive and highly reliable diagnostic procedures in identifying endoductal lesions.

Our study presented two major limitations: The retrospective nature and small sample of patients undergoing an invasive procedure with DBT-technique. Prospective studies can help resolve the former.

\section{Conclusion}

Diagnostic algorithm in PND including cytological examination, mammography, and ultrasound whole breast is recommended as the first-line investigation. In our study, we recommend intraductal invasive procedures as an additional tool to identify intraductal breast lesions when cytological outcomes were "bloody" or "atypical/suspected. Because of its efficacy and precision, state-of-the-art ductography should be an essential diagnostic modality for preoperative planning to define the precise location of endoductal breast lesions and the extension of multiple coexisting lesions. Digital imaging and new technologies such as 3D-tomosynthesis will can lead to a renaissance of breast invasive imaging. 


\section{Conflicts of Interest}

The authors declare no conflicts of interest regarding the publication of this paper.

\section{References}

[1] Rissanen, T., Reinikainen, H. and Apaja-Sarkkinen, M. (2007) Breast Sonography in Localizing the Cause of Nipple Discharge: Comparison with Galactography in 52 Patients. Journal of Ultrasound in Medicine, 26, 1031-1039. https://doi.org/10.7863/jum.2007.26.8.1031

[2] Srinivasan, A., Nia, E., Gupta, M., Sun, J. and Leung, J.W. (2019) Retrospective Statistical Analysis on the Diagnostic Value of Ductography Based on Lesion Pathology in Patients Presenting with Nipple Discharge. The Breast Journal, 25, 585-589. https://doi.org/10.1111/tbj.13298

[3] Baydoun, S., Gonzalez, P., Whitman, G.J., Dryden, M., Xi, Y. and Dogan, B. (2019) Is Ductography Still Warranted in the 21st Century? The Breast Journal, 25, 654-662. https://doi.org/10.1111/tbj.13302

[4] Expert Panel on Breast Imaging, Lee, S.J., Trikha, S., Moy, L., Baron, P., diFlorio, R.M., Green, E.D., et al. (2017) ACR Appropriateness Criteria ${ }^{\circledR}$ Evaluation of Nipple Discharge. Journal of the American College of Radiology, 14, S138-S153. https://doi.org/10.1016/j.jacr.2017.01.030

[5] Adepoju, L.J., Chun, J., El-Tamer, M., Ditkoff, B.A., Schnabel, F. and Joseph, K.A. (2005) The Value of Clinical Characteristics and Breast-Imaging Studies in Predicting a Histopathologic Diagnosis of Cancer or High-Risk Lesion in Patients with Spontaneous Nipple Discharge. The American Journal of Surgery, 190, 644-646. https://doi.org/10.1016/j.amjsurg.2005.06.032

[6] Lippa, N., Hurtevent-Labrot, G., Ferron, S. and Boisserie-Lacroix, M. (2015) Nipple Discharge: The Role of Imaging. Diagnostic and Interventional Imaging, 96, 1017-1032. https://doi.org/10.1016/j.diii.2015.07.004

[7] Bahl, M., Baker, J.A., Greenup, R.A. and Ghate, S.V. (2015) Diagnostic Value of U1trasound in Female Patients with Nipple Discharge. American Journal of Roentgenology, 205, 203-208. https://doi.org/10.2214/AJR.14.13354

[8] Kooistra, B.W., Wauters, C., van de Ven, S. and Strobbe, L. (2009) The Diagnostic Value of Nipple Discharge Cytology in 618 Consecutive Patients. European Journal of Surgical Oncology, 35, 573-577. https://doi.org/10.1016/j.ejso.2008.09.009

[9] Moriarty, A.T., Schwartz, M.R., Laucirica, R., Booth, C.N., Auger, M., Thomas, N.E., et al. (2013) Cytology of Spontaneous Nipple Discharge-Is It Worth It? Performance of Nipple Discharge Preparations in the College of American Pathologists Interlaboratory Comparison Program in Nongynecologic Cytopathology. Archives of Pathology \& Laboratory Medicine, 137, 1039-1042.

https://doi.org/10.5858/arpa.2012-0231-CP

[10] Istomin, A., Masarwah, A., Pitkanen, M., Joukainen, S., Sutela, A., Vanninen, R., et al. (2018) Galactography Is Not an Obsolete Investigation in the Evaluation of $\mathrm{Pa}$ thological Nipple Discharge. PLoS ONE, 13, e0204326.

https://doi.org/10.1371/journal.pone.0204326

[11] Tabar, L., Dean, P.B. and Pentek, Z. (1983) Galactography: The Diagnostic Procedure of Choice for Nipple Discharge. Radiology, 149, 31-38.

https://doi.org/10.1148/radiology.149.1.6611939

[12] Patel, B.K., Falcon, S. and Drukteinis, J. (2015) Management of Nipple Discharge 
and the Associated Imaging Findings. The American Journal of Medicine, 128, 353-360. https://doi.org/10.1016/j.amjmed.2014.09.031

[13] Schulz-Wendtland, R., Preuss, C., Fasching, P.A., Loehberg, C.R., Lux, M.P., Emons, J., et al. (2018) Galactography with Tomosynthesis Technique (Galactomosynthesis) -Renaissance of a Method? Geburtshilfe und Frauenheilkunde, 78, 493-498. https://doi.org/10.1055/a-0594-2277

[14] Rafferty, E.A., Durand, M.A., Conant, E.F., Copit, D.S., Friedewald, S.M., Plecha, D.M., et al. (2016) Breast Cancer Screening Using Tomosynthesis and Digital Mammography in Dense and Nondense Breasts. JAMA, 315, 1784-1786. https://doi.org/10.1001/jama.2016.1708

[15] Lubina, N., Schedelbeck, U., Roth, A., Weng, A.M., Geissinger, E., Hönig, A., et al. (2015) 3.0 Tesla Breast Magnetic Resonance Imaging in Patients with Nipple Discharge When Mammography and Ultrasound Fail. European Radiology, 25, 1285-1293. https://doi.org/10.1007/s00330-014-3521-2

[16] Bahl, M., Baker, J.A., Greenup, R.A. and Ghate, S.V. (2015) Evaluation of Pathologic Nipple Discharge: What Is the Added Diagnostic Value of MRI? Annals of Surgical Oncology, 22, S435-S441. https://doi.org/10.1245/s10434-015-4792-9

[17] Dhillon, G.S., Bell, N., Ginat, D.T., Levit, A., Destounis, S. and O'Connell, A. (2011) Breast MR Imaging: What the Radiologist Needs to Know. Journal of Clinical Imaging Science, 1, 48. https://doi.org/10.4103/2156-7514.85655

[18] D’Orsi, C.J., Sickles, E.A. and Mendelson, E.B. (2013) ACR BI-RADS ${ }^{\circledast}$ Atlas, Breast Imaging Reporting and Data System. American College of Radiology, Reston.

[19] Lamont, J.P., Dultz, R.P., Kuhn, J.A., Grant, M.D. and Jones, R.C. (2000) Galactography in Patients with Nipple Discharge. Proceedings (Baylor University. Medical Center), 13, 214-216. https://doi.org/10.1080/08998280.2000.11927676

[20] Yoon, J.H., Yoon, H., Kim, E.K., Moon, H.J., Park, Y.V. and Kim, M.J. (2017) Ultrasonographic Evaluation of Women with Pathologic Nipple Discharge. Ultrasonography, 36, 310-320. https://doi.org/10.14366/usg.17013

[21] Berná-Serna, J.D., Redondo, M.V., Durán, I. and Berná-Mestre, J.D. (2008) Galactography without Discomfort Using Lidocaine/Prilocaine Anesthetic Cream. Acta Radiologica, 49, 22-24. https://doi.org/10.1080/02841850701630318

[22] Berna-Serna, J.D., Torres-Ales, C., Berna-Mestre, J.D., Sola-Perez, J. and Canteras-Jordana, M. (2010) Galactography: An Application of the Galactogram Imaging Classification System (GICS). Acta Radiologica, 51, 128-136. https://doi.org/10.3109/02841850903436659

[23] Sardanelli, F., Boetes, C., Borisch, B., Decker, T., Federico, M., Gilbert, F.J., et al. (2010) Magnetic Resonance Imaging of the Breast: Recommendations from the EUSOMA Working Group. European Journal of Cancer, 46, 1296-1316. https://doi.org/10.1016/j.ejca.2010.02.015

[24] Lorenzon, M., Zuiani, C., Linda, A., Londero, V., Girometti, R. and Bazzocchi, M. (2011) Magnetic Resonance Imaging in Patients with Nipple Discharge: Should We Recommend It? European Radiology, 21, 899-907. https://doi.org/10.1007/s00330-010-2009-y

[25] Berger, N., Luparia, A., Di Leo, G., Carbonaro, L.A., Trimboli, R.M., Ambrogi, F., et al. (2017) Diagnostic Performance of MRI versus Galactography in Women with Pathologic Nipple Discharge: A Systematic Review and Meta-Analysis. American Journal of Roentgenology, 209, 465-471. https://doi.org/10.2214/AJR.16.16682 